\title{
Plasminogen activator inhibitor-1 4G/5G polymorphism and retinopathy risk in type 2 diabetes: a meta-analysis
}

Tengyue Zhang ${ }^{1,2,3{ }^{\dagger}}$, Chong Pang ${ }^{4+}$, Ningdong $\mathrm{Li}^{2}$, Elaine Zhou ${ }^{5}$ and Kanxing Zhao ${ }^{2^{*}}$

\begin{abstract}
Background: Mounting evidence has suggested that plasminogen activator inhibitor-1 (PAl-1) is a candidate for increased risk of diabetic retinopathy. Studies have reported that insertion/deletion polymorphism in the PAl-1 gene may influence the risk of this disease. To comprehensively address this issue, we performed a meta-analysis to evaluate the association of PAl-1 4G/5G polymorphism with diabetic retinopathy in type 2 diabetes.

Methods: Data were retrieved in a systematic manner and analyzed using Review Manager and STATA Statistical Software. Crude odds ratios (ORs) with 95\% confidence intervals (Cls) were used to assess the strength of associations.

Results: Nine studies with 1, 217 cases and 1,459 controls were included. Allelic and genotypic comparisons between cases and controls were evaluated. Overall analysis suggests a marginal association of the 4G/5G polymorphism with diabetic retinopathy (for $4 \mathrm{G}$ versus $5 \mathrm{G}$ : OR $1.13,95 \% \mathrm{Cl} 1.01$ to 1.26 ; for $4 \mathrm{G} / 4 \mathrm{G}$ versus 5G/5G: OR $1.30,95 \% \mathrm{Cl} 1.04$ to 1.64 ; for $4 \mathrm{G} / 4 \mathrm{G}$ versus $5 \mathrm{G} / 5 \mathrm{G}+4 \mathrm{G} / 5 \mathrm{G}$ : OR $1.26,95 \% \mathrm{Cl} 1.05$ to 1.52 ). In subgroup analysis by ethnicity, we found an association among the Caucasian population (for $4 \mathrm{G}$ versus $5 \mathrm{G}$ : OR $1.14,95 \% \mathrm{Cl} 1.00$ to 1.30; for $4 \mathrm{G} / 4 \mathrm{G}$ versus $5 \mathrm{G} / 5 \mathrm{G}$ : OR $1.33,95 \% \mathrm{Cl} 1.02$ to 1.74 ; for $4 \mathrm{G} / 4 \mathrm{G}$ versus $5 \mathrm{G} / 5 \mathrm{G}+4 \mathrm{G} / 5 \mathrm{G}$ : OR $1.41,95 \% \mathrm{Cl} 1.13$ to 1.77 ). When stratified by the average duration of diabetes, patients with diabetes histories longer than 10 years have an elevated susceptibility to diabetic retinopathy than those with shorter histories (for 4G/4G versus 5G/5G: OR 1.47, $95 \% \mathrm{Cl} 1.08$ to 2.00). We also detected a higher risk in hospital-based studies (for $4 \mathrm{G} / 4 \mathrm{G}$ versus $5 \mathrm{G} / 5 \mathrm{G}+4 \mathrm{G} / 5 \mathrm{G}$ : OR $1.27,95 \% \mathrm{Cl} 1.02$ to 1.57$)$.
\end{abstract}

Conclusions: The present meta-analysis suggested that $4 \mathrm{G} / 5 \mathrm{G}$ polymorphism in the PAl-1 gene potentially increased the risk of diabetic retinopathy in type 2 diabetes and showed a discrepancy in different ethnicities. A higher susceptibility in patients with longer duration of diabetes (more than 10 years) indicated a geneenvironment interaction in determining the risk of diabetic retinopathy.

Keywords: Diabetic retinopathy, meta-analysis, PAI-1, polymorphism, type 2 diabetes

\section{Background}

Diabetic retinopathy (DR), the leading cause of blindness in the working population, is associated with a strong genetic predisposition, highlighted by the familial clustering of DR $[1,2]$. Several gene polymorphisms are associated with DR, such as in the methylenetetrahydrofolate

\footnotetext{
* Correspondence: zkx4260@vip.163.com

+ Contributed equally

${ }^{2}$ Tianjin Eye Hospital, Tianjin Key Laboratory of Ophthalmology and Visual Science, Tianjin Eye Institute, Clinical College of Ophthalmology Tianjin

Medical University, 4 Gansu Road, Tianjin, 300020, PR China

Full list of author information is available at the end of the article
}

reductase gene, endothelial nitric oxide synthase gene, manganese superoxide dismutase gene, vascular endothelial growth factor gene, receptor for advanced glycation end products gene, aldose reductase 2 gene and P-selectin gene, among others [3-9]. Plasminogen activator inhibitor1 (PAI-1), the most important in vivo inhibitor of plasminogen activation, has also been implicated in DR. In addition to being involved in tissue repair and remodeling, PAI-1 plays a critical role in the regulation of intravascular fibrinolysis. In patients with type 2 diabetes (T2D), impaired fibrinolysis is involved in the pathogenesis of DR

\section{Biomed Central}


[10]. Increased PAI-1 expression has been associated with matrix accumulation [11] and the development of basement membrane thickening and pericyte loss, which are regarded as the earliest retinal pathohistological changes in DR in transgenic mice $[10,12]$. PAI-1 activity, which is affected by $P A I-1$ gene polymorphisms and metabolic determinants, is also elevated in DR [13]. Compared with other PAI- 1 variants, the most significant variation in PAI1 expression resides in a common single-base-pair guanine insertion/deletion polymorphism (4G/5G) within the promoter region of the PAI-1 gene at nucleotide position -675 $[11,14]$. Unlike the $5 \mathrm{G}$ allele that binds a transcription repressor protein, resulting in low PAI-1 expression, the 4G allele does not bind a transcription repressor, thus conferring a 'high PAI-1 expressor' nature to the allele [15].

Considering the potential influence on the individual risk for DR due to the insertion-deletion mutation of -675 4G/5G, many studies have explored the association between PAI-1 4G/5G and DR risk [16-24]. However, individual studies yielded inconsistent and even conflicting findings, which might be caused by the limitation of individual studies. To shed light on these contradictory results and to get a more precise evaluation of this association, we performed a meta-analysis of nine published case-control studies covering 1, 217 cases and 1, 459 controls.

\section{Methods}

\section{Search strategy}

In this meta-analysis, a comprehensive literature research of the US National Library of Medicine's PubMed database (to 1 May 2012) was conducted using research terms including 'plasminogen activator inhibitor-1', 'PAI1', '4G/5G', 'polymorphism', 'type 2 diabetes', 'diabetic retinopathy' and the combined phrases to obtain all genetic studies on the relationship between PAI-1 4G/5G polymorphism and DR risk. There was no language limitation. We also hand-searched references of original studies or review articles on this topic to identify additional studies. The following criteria were used to select the eligible studies: they must be case-control studies on the association between $P A I-14 \mathrm{G} / 5 \mathrm{G}$ polymorphism and DR; and they must contain detailed and correct numbers of different genotypes for estimating an odds ratio (OR) with a $95 \%$ confidence interval (CI). When several publications reported on the same population data, the largest or most complete study was chosen. As a result, nine case-control studies were included in our meta-analysis.

\section{Data extraction}

Two investigators independently assessed the articles for inclusion or exclusion, resolved disagreements, and attained consistency. For each eligible study, the following information was recorded: the first author's name, the year of publication, country of origin, ethnicity, total number of patients with DR and number of participants without DR (DWR) as well as the DR/DWR distribution in each PAI-1 genotype. Different ethnicities were categorized as Caucasian, Asian and Pima Indian. Sources of control were divided into population-based and hospital-based controls. The average duration of diabetes was separated into longer and shorter than 10 years.

\section{Statistical analysis}

The strength of the relationship between PAI-1 4G/5G polymorphism and DR risk was assessed by calculating pooled ORs with $95 \%$ CIs. We evaluated the risk using the codominant model (4G/4G versus $5 \mathrm{G} / 5 \mathrm{G}$; $4 \mathrm{G} / 5 \mathrm{G}$ versus $5 \mathrm{G} / 5 \mathrm{G})$, the dominant model $(4 \mathrm{G} / 4 \mathrm{G}+4 \mathrm{G} / 5 \mathrm{G}$ versus $5 \mathrm{G} /$ $5 G$ ) and the recessive model (4G/4G versus $4 \mathrm{G} / 5 \mathrm{G}+5 \mathrm{G} /$ $5 G)$. Between-study heterogeneity was evaluated by $\chi^{2}-$ based Q-test [25] and was considered significant if $P<$ 0.10 , in which case the random-effects model (the DerSimonian and Laird method [26]) was used to pool the data. If $P>0.10$, the fixed-effects model (the Mantel-Haenszel method [27]) was selected. These two models provided similar results when between-studies heterogeneity was absent. Begg's funnel plot, a scatter plot of effect against a measure of study size, was generated as a visual aid for detecting bias or systematic heterogeneity [28]. Publication bias was assessed by the linear regression asymmetry test by Egger et al. $(P<0.05$ considered representative of statistical significance [29]). Studies were categorized into subgroups based on ethnicity, average diabetes duration, and source of control. Hardy-Weinberg equilibrium was tested by the $\chi^{2}$ test. All statistical analyses were performed in Review Manager (v.5.0; Oxford, England) and STATA Statistical Software (v.10.0; StataCorp, LP, College Station, TX). A two sided $P$-value $<0.05$ was considered statistically significant.

\section{Results \\ Eligible studies}

In total, nine case-control studies including 1, 217 cases and 1, 459 controls were selected in our meta-analysis. A flow chart of the literature search, according to the Preferred Reporting Items for Systematic Reviews and MetaAnalyses guidelines [30], is shown in Figure 1. The main characteristics of these studies are shown in Table 1. Among these eligible publications, there were five studies of Caucasians, three studies of Asians and one study of Pima Indians. Four studies in which the average diabetes duration in all the subgroups was longer than 10 years were enrolled together and compared with the other five studies in which the diabetes duration was shorter than 10 years. There were two population-based studies and seven hospital-based studies. All studies used PCR methods for 


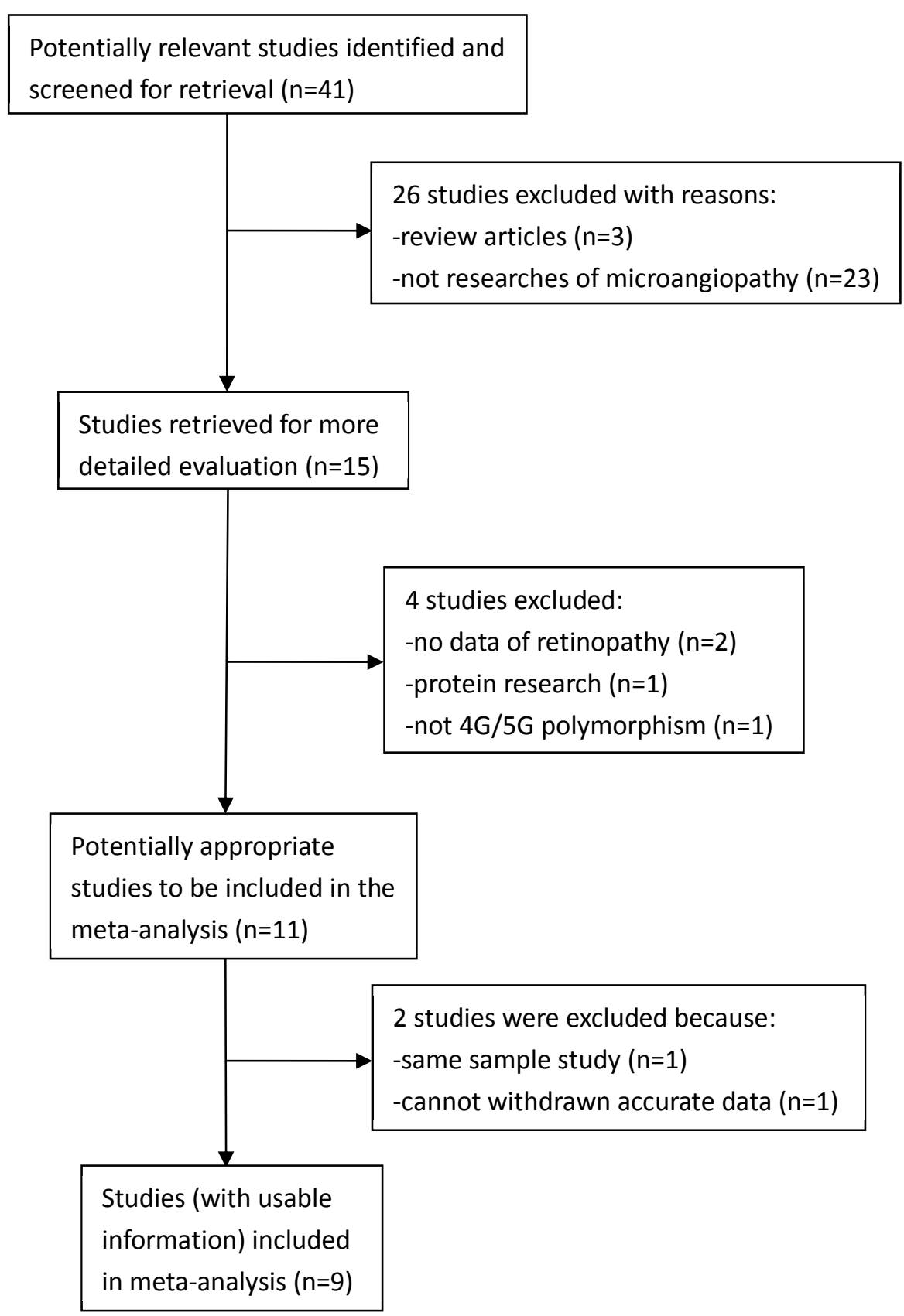

Figure 1 Flow chart of included studies.

genotyping. The genotype distributions in the controls of all studies were in agreement with Hardy-Weinberg equilibrium. Thus, the sensitivity analysis was not performed in this study.

\section{Meta-analysis}

The main results of this meta-analysis and the heterogeneity test are shown in Table 2 . Overall, we found a marginally statistical significant association between 4G/ $4 \mathrm{G}$ and DR risk in overall population (for $4 \mathrm{G} / 4 \mathrm{G}$ versus 5G/5G: OR 1.30, 95\%CI 1.04 to 1.64, Figure 2; for 4G/ $4 \mathrm{G}$ versus $5 \mathrm{G} / 5 \mathrm{G}+4 \mathrm{G} / 5 \mathrm{G}$ : OR 1.26 , $95 \% \mathrm{CI} 1.05$ to $1.52)$. In the subgroup analysis by ethnicity, significantly increased risks were observed among the Caucasian population (for $4 \mathrm{G} / 4 \mathrm{G}$ versus $5 \mathrm{G} / 5 \mathrm{G}$ : OR 1.33 , $95 \% \mathrm{CI}$ 1.02 to 1.74 ; for $4 \mathrm{G} / 4 \mathrm{G}$ versus $5 \mathrm{G} / 5 \mathrm{G}+4 \mathrm{G} / 5 \mathrm{G}$ : OR 
Table 1 Characteristics of case-control studies included in the meta-analysis.

\begin{tabular}{|c|c|c|c|c|c|c|c|c|c|c|c|c|}
\hline \multirow[t]{3}{*}{ First author } & \multirow[t]{3}{*}{ Year } & \multirow[t]{3}{*}{ Country } & \multirow[t]{3}{*}{ Ethnicity } & \multicolumn{6}{|c|}{ Distribution of PAI genotypes } & \multirow{3}{*}{$\begin{array}{l}\text { Sample Size } \\
\text { (DR/DWR) }\end{array}$} & \multirow[t]{3}{*}{ HWE } & \multirow{3}{*}{$\begin{array}{l}\text { Reference } \\
\text { Number }\end{array}$} \\
\hline & & & & \multicolumn{2}{|c|}{ 4G/4G } & \multicolumn{2}{|c|}{ 4G/5G } & \multicolumn{2}{|c|}{$5 \mathrm{G} / 5 \mathrm{G}$} & & & \\
\hline & & & & DR & DWR & DR & DWR & DR & DWR & & & \\
\hline Nagi & 1997 & U.S.A. & Pima Indian & 14 & 18 & 44 & 45 & 12 & 39 & $70 / 102$ & 0.43 & [19] \\
\hline Broch & 1998 & Spain & Caucasian & 17 & 19 & 46 & 48 & 19 & 28 & $82 / 95$ & 0.85 & [21] \\
\hline Wong & 2000 & China & East Asian & 31 & 16 & 38 & 28 & 15 & 13 & $84 / 57$ & 0.91 & [23] \\
\hline Santos & 2003 & Brazil & Caucasian & 24 & 22 & 41 & 59 & 34 & 30 & $99 / 111$ & 0.47 & {$[16]$} \\
\hline Globocnik-Petrovic & 2003 & Slovenia & Caucasian & 39 & 25 & 58 & 40 & 27 & 15 & $124 / 80$ & 0.89 & [18] \\
\hline Liu & 2004 & China & East Asian & 15 & 27 & 26 & 49 & 15 & 15 & $56 / 91$ & 0.36 & [24] \\
\hline Zietz & 2004 & Germany & Caucasian & 48 & 112 & 55 & 173 & 28 & 73 & $131 / 358$ & 0.68 & [20] \\
\hline Murata & 2004 & Japan & East Asian & 78 & 43 & 86 & 35 & 24 & 14 & 188/92 & 0.14 & [22] \\
\hline Ezzidi & 2009 & Tunisia & Caucasian & 77 & 54 & 167 & 242 & 139 & 177 & $383 / 473$ & 0.56 & [17] \\
\hline
\end{tabular}

DR: diabetic retinopathy; DWR: diabetes without retinopathy; HWE: Hardy-Weinberg equilibrium.

Table 2 Summary of odds ratios and 95\% confidence intervals of PAI-1 4G/5G polymorphism and diabetic retinopathy risk.

\begin{tabular}{|c|c|c|c|c|}
\hline Comparisons & $\begin{array}{l}\text { Number } \\
\text { of studies }\end{array}$ & OR $(95 \% \mathrm{Cl})$ & $P^{a}$ & $\begin{array}{l}\text { Alleles/genotypes } \\
\text { (DR/DWR) }\end{array}$ \\
\hline \multicolumn{5}{|l|}{$4 \mathrm{G} / 4 \mathrm{G}$ versus $5 \mathrm{G} / 5 \mathrm{G}$} \\
\hline Total & 9 & $1.30(1.04,1.64)$ & 0.27 & 1, $396(656 / 740)$ \\
\hline \multicolumn{5}{|l|}{ Source of control } \\
\hline Population based & 2 & $1.37(0.85,2.20)$ & 0.15 & $344(102 / 242)$ \\
\hline Hospital based & 7 & $1.28(0.99,1.67)$ & 0.26 & 1, $052(554 / 498)$ \\
\hline \multicolumn{5}{|l|}{ Ethnicity } \\
\hline Caucasian & 5 & $1.33(1.02,1.74)$ & 0.36 & $1,007(452 / 555)$ \\
\hline Asian & 3 & $1.00(0.61,1.65)$ & 0.27 & $306(178 / 128)$ \\
\hline \multicolumn{5}{|l|}{ Average diabetes duration } \\
\hline Longer than 10 years & 4 & $1.47(1.08,2.00)$ & 0.33 & $787(430 / 357)$ \\
\hline Shorter than 10 years & 5 & $1.13(0.80,1.58)$ & 0.27 & $609(226 / 383)$ \\
\hline \multicolumn{5}{|l|}{$4 \mathrm{G} / 5 \mathrm{G}$ versus $5 \mathrm{G} / 5 \mathrm{G}$} \\
\hline Total & 9 & $1.01(0.74,1.37)$ & 0.04 & 1, $970(874 / 1,123)$ \\
\hline \multicolumn{5}{|l|}{ Source of control } \\
\hline Population based & 2 & $1.58(0.42,5.88)$ & $0.00^{\mathbf{b}}$ & $469(139 / 330)$ \\
\hline Hospital based & 7 & $0.90(0.72,1.11)$ & 0.39 & $1,528(735 / 793)$ \\
\hline \multicolumn{5}{|l|}{ Ethnicity } \\
\hline Caucasian & 5 & $0.87(0.70,1.08)$ & 0.55 & $1,499(614 / 885)$ \\
\hline Asian & 3 & $0.99(0.61,1.60)$ & 0.22 & $358(204 / 154)$ \\
\hline \multicolumn{5}{|l|}{ Average diabetes duration } \\
\hline Longer than 10 years & 4 & $0.94(0.73,1.20)$ & 0.62 & $1,118(554 / 564)$ \\
\hline Shorter than 10 years & 5 & $1.03(0.57,1.85)$ & $0.00^{\mathbf{b}}$ & $879(320 / 559)$ \\
\hline \multicolumn{5}{|c|}{$4 \mathrm{G} / 4 \mathrm{G}$ versus $5 \mathrm{G} / 5 \mathrm{G}+4 \mathrm{G} / 5 \mathrm{G}$} \\
\hline Total & 9 & $1.26(1.05,1.52)$ & 0.25 & $2,676(1,217 / 1,459)$ \\
\hline \multicolumn{5}{|l|}{ Source of control } \\
\hline Population based & 2 & $1.25(0.86,1.80)$ & 0.85 & $661(201 / 460)$ \\
\hline Hospital based & 7 & $1.27(1.02,1.57)$ & 0.12 & 2, $015(1,016 / 999)$ \\
\hline \multicolumn{5}{|l|}{ Ethnicity } \\
\hline Caucasian & 5 & $1.41(1.13,1.77)$ & 0.29 & $1,936(819 / 1117)$ \\
\hline Asian & 3 & $0.96(0.67,1.37)$ & 0.37 & $568(328 / 240)$ \\
\hline \multicolumn{5}{|l|}{ Average diabetes duration } \\
\hline Longer than 10 years & 4 & $1.26(0.80,1.98)$ & $0.03^{\mathbf{b}}$ & $1,481(779 / 702)$ \\
\hline Shorter than 10 years & 5 & $1.16(0.89,1.53)$ & 0.92 & $1,195(438 / 757)$ \\
\hline
\end{tabular}


Table 2 Summary of odds ratios and 95\% confidence intervals of PAI-1 4G/5G polymorphism and diabetic retinopathy risk. (Continued)

\begin{tabular}{|c|c|c|c|c|}
\hline \multicolumn{5}{|c|}{$4 G / 4 G+4 G / 5 G$ versus $5 G / 5 G$} \\
\hline Total & 9 & $1.07(0.82,1.39)$ & $0.09^{\mathbf{b}}$ & $2,679(1,217 / 1,459)$ \\
\hline \multicolumn{5}{|l|}{ Source of control } \\
\hline Population based & 2 & $1.62(0.52,5.03)$ & $0.01^{\mathbf{b}}$ & $661(201 / 460)$ \\
\hline Hospital based & 7 & $0.99(0.81,1.22)$ & 0.46 & $2,015(1,016 / 999)$ \\
\hline \multicolumn{5}{|l|}{ Ethnicity } \\
\hline Caucasian & 5 & $0.99(0.81,1.21)$ & 0.62 & $1,936(819 / 1,117)$ \\
\hline Asian & 3 & $0.98(0.62,1.54)$ & 0.22 & $568(328 / 240)$ \\
\hline \multicolumn{5}{|l|}{ Average diabetes duration } \\
\hline Longer than 10 years & 4 & $1.06(0.84,1.34)$ & 0.81 & 1, $481(779 / 702)$ \\
\hline Shorter than 10 years & 5 & $1.08(0.64,1.81)$ & $0.01^{\mathbf{b}}$ & $1,195(438 / 757)$ \\
\hline \multicolumn{5}{|l|}{$4 \mathrm{G}$ versus $5 \mathrm{G}$} \\
\hline Total & 9 & $1.13(1.01,1.26)$ & 0.31 & $5,364(2,446 / 2,918)$ \\
\hline \multicolumn{5}{|l|}{ Source of control } \\
\hline Population based & 2 & $1.23(0.97,1.56)$ & 0.15 & 1, $322(402 / 920)$ \\
\hline Hospital based & 7 & $1.10(0.97,1.25)$ & 0.35 & $4,042(2,044 / 1,998)$ \\
\hline \multicolumn{5}{|l|}{ Ethnicity } \\
\hline Caucasian & 5 & $1.14(1.00,1.30)$ & 0.57 & $3,884(1,650 / 2,234)$ \\
\hline Asian & 3 & $0.97(0.76,1.25)$ & 0.27 & $1,136(656 / 480)$ \\
\hline \multicolumn{5}{|l|}{ Average diabetes duration } \\
\hline Longer than 10 years & 4 & $1.14(0.98,1.33)$ & 0.42 & $2,962(15,58 / 1,404)$ \\
\hline Shorter than 10 years & 5 & $1.11(0.94,1.32)$ & 0.16 & $2,402(888 / 1,514)$ \\
\hline
\end{tabular}

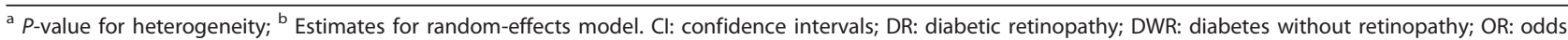
ratio.

$1.41,95 \%$ CI 1.13 to 1.77 , Figure 3$)$. In the stratified analysis by average diabetes duration, the PAI-1 variation was found associated with elevated DR risk in patients with a duration of diabetes longer than 10 years (for 4G/4G versus 5G/5G: OR 1.47 , 95\%CI 1.08 to 2.00 , Figure 4). We also detected an increasing risk in hospital-based studies (for $4 \mathrm{G} / 4 \mathrm{G}$ versus $5 \mathrm{G} / 5 \mathrm{G}+4 \mathrm{G} / 5 \mathrm{G}$ : OR 1.27 , 95\%CI 1.02 to 1.57 , Figure 5).

\section{Publication bias}

The potential presence of publication bias was evaluated quantitatively by Begg's funnel plot and Egger's test. The Begg's funnel plot appeared symmetric. The Egger's test supported that there was no significant statistical evidence of publication bias for any of the four genetic models (for 4G/4G versus $5 \mathrm{G} / 5 \mathrm{G}: P=0.281$; for $4 \mathrm{G} / 4 \mathrm{G}$ versus $5 \mathrm{G} / 5 \mathrm{G}$ + 4G/5G: $P=0.169$; for $4 \mathrm{G} / 5 \mathrm{G}$ versus $5 \mathrm{G} / 5 \mathrm{G}: P=0,462$;

\begin{tabular}{|c|c|c|c|c|c|c|c|c|}
\hline Studv or Subgroup & $\begin{array}{r}\text { Case } \\
\text { Events }\end{array}$ & Total & \multicolumn{2}{|c|}{ Control } & Weight & $\begin{array}{l}\text { Odds Ratio } \\
\text { M-H. Fixed. 95\% Cl Year }\end{array}$ & \multicolumn{2}{|c|}{$\begin{array}{c}\text { Odds Ratio } \\
\text { M-H. Fixed. } 95 \% \mathrm{Cl}\end{array}$} \\
\hline Nagi & 14 & 26 & 18 & 57 & $4.1 \%$ & $2.53[0.98,6.55] 1997$ & & \\
\hline Broch & 17 & 36 & 19 & 47 & $6.8 \%$ & $1.32[0.55,3.17] 1998$ & & \\
\hline Wong & 31 & 46 & 16 & 29 & $5.0 \%$ & $1.68[0.64,4.37] 2000$ & & \\
\hline Globocnik-petrovic & 39 & 66 & 25 & 40 & $9.9 \%$ & $0.87[0.39,1.94] 2003$ & & \\
\hline Santos & 24 & 58 & 22 & 52 & $10.6 \%$ & $0.96[0.45,2.06] 2003$ & & \\
\hline Zietz & 48 & 76 & 112 & 185 & $18.7 \%$ & $1.12[0.64,1.94] 2004$ & & \\
\hline Liu & 15 & 30 & 27 & 42 & $8.8 \%$ & $0.56[0.21,1.44] 2004$ & & \\
\hline Murata & 78 & 102 & 43 & 57 & $10.1 \%$ & $1.06[0.50,2.26] 2004$ & & \\
\hline Ezzidi & 77 & 216 & 54 & 231 & $26.1 \%$ & $1.82[1.20,2.74] 2009$ & & - \\
\hline Total $(95 \% \mathrm{Cl})$ & & 656 & & 740 & $100.0 \%$ & $1.30[1.04,1.64]$ & & \\
\hline Total events & 343 & & 336 & & & & & \\
\hline $\begin{array}{l}\text { Heterogeneity: } \mathrm{Chi}^{2}= \\
\text { Test for overall effect: }\end{array}$ & $\begin{array}{l}86, \mathrm{df}=8 \\
=2.26(\mathrm{P}\end{array}$ & $\begin{array}{l}(P=0 \\
=0.02\end{array}$ & $\begin{array}{l}\text { 2) } 27) ; 1^{2}= \\
\text { 2) }\end{array}$ & $19 \%$ & & & 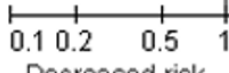 & 12 \\
\hline
\end{tabular}

Figure 2 Odds ratios of diabetic retinopathy associated with PAl-1 polymorphism (4G/4G versus 5G/5G) in overall studies. 


\begin{tabular}{|c|c|c|c|c|c|c|c|}
\hline Studv or Subgroup & $\begin{array}{l}\text { Case } \\
\text { Events }\end{array}$ & Total & $\begin{array}{l}\text { Contre } \\
\text { Events }\end{array}$ & $\begin{array}{l}\text { ol } \\
\text { Total }\end{array}$ & Weight & $\begin{array}{l}\text { Odds Ratio } \\
\text { M-H. Fixed. } 95 \% \mathrm{Cl} \text { Year }\end{array}$ & $\begin{array}{l}\text { Odds Ratio } \\
\text { M.-H. Fixed. } 95 \% \mathrm{Cl}\end{array}$ \\
\hline Broch & 17 & 82 & 19 & 95 & $11.0 \%$ & $1.05[0.50,2.18] 1998$ & \\
\hline Santos & 24 & 99 & 22 & 111 & $12.4 \%$ & $1.29[0.67,2.49] 2003$ & \\
\hline Globocnik-petrovic & 39 & 124 & 25 & 80 & $16.4 \%$ & $1.01[0.55,1.85] 2003$ & \\
\hline Zietz & 48 & 131 & 112 & 358 & $29.9 \%$ & $1.27[0.83,1.93] 2004$ & \\
\hline Ezzidi & 77 & 383 & 54 & 473 & $30.4 \%$ & $1.95[1.34,2.85] 2009$ & $=$ \\
\hline Total $(95 \% \mathrm{Cl})$ & & 819 & & 1117 & $100.0 \%$ & $1.41[1.13,1.77]$ & \\
\hline Total events & 205 & & 232 & & & & \\
\hline \multicolumn{7}{|c|}{$\begin{array}{l}\text { Heterogeneity: } \mathrm{Ch}^{2}=4.96, \mathrm{df}=4(\mathrm{P}=0.29) ; \mathrm{I}^{2}=19 \% \\
\text { Test for owerall effect: } Z=3.01(P=0.003)\end{array}$} & 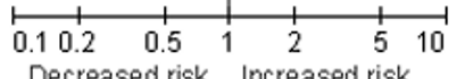 \\
\hline
\end{tabular}

Figure 3 Odds ratios of diabetic retinopathy associated with PAl-1 polymorphism (4G/4G versus 4G/5G + 5G/5G) in Caucasian populations.

\begin{tabular}{|c|c|c|c|c|c|c|c|c|}
\hline Studv or Subqroup & $\begin{array}{l}\text { Case } \\
\text { Events }\end{array}$ & Total & \multicolumn{2}{|c|}{ Control } & Weight & $\begin{array}{l}\text { Odds Ratio } \\
\text { M-H.Fixed. } 95 \% \text { Cl Year }\end{array}$ & \multicolumn{2}{|c|}{$\begin{array}{c}\text { Odds Ratio } \\
\text { M.H. Fixed. } 95 \% \mathrm{Cl}\end{array}$} \\
\hline Wong & 31 & 46 & 16 & 29 & $9.7 \%$ & $1.68[0.64,4.37] 2000$ & & \\
\hline Globocnik-petrowic & 39 & 66 & 25 & 40 & $19.4 \%$ & $0.87[0.39,1.94] 2003$ & & \\
\hline Murata & 78 & 102 & 43 & 57 & $19.8 \%$ & $1.06[0.50,2.26] 2004$ & & \\
\hline Ezzidi & 77 & 216 & 54 & 231 & $51.1 \%$ & $1.82[1.20,2.74] 2009$ & & \\
\hline Total $(95 \% \mathrm{Cl})$ & & 430 & & 357 & $100.0 \%$ & $1.47[1.08,2.00]$ & & \\
\hline Total events & 225 & & 138 & & & & & \\
\hline $\begin{array}{l}\text { Heterogeneity: } \mathrm{Chi}^{2}= \\
\text { Test for overall effect: }\end{array}$ & $\begin{array}{l}3.45, \mathrm{df}=3 \\
Z=2.43(\mathrm{P}\end{array}$ & $\begin{array}{l}(P=0 \\
=0.0\end{array}$ & $\begin{array}{l}\text { 1) } 33 ; 1^{2}= \\
\end{array}$ & $13 \%$ & & & 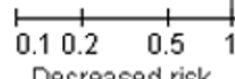 & $\begin{array}{llll}1 & 2 & 5 & 10\end{array}$ \\
\hline
\end{tabular}

Figure 4 Odds ratios of diabetic retinopathy associated with PAl-1 polymorphism (4G/4G versus 5G/5G) in average diabetes duration $>10$ years.

\begin{tabular}{|c|c|c|c|c|c|c|c|}
\hline Studv or Subgroup & $\begin{array}{l}\text { Case } \\
\text { Events }\end{array}$ & Total & $\begin{array}{l}\text { Contro } \\
\text { Events }\end{array}$ & $\begin{array}{l}\text { ol } \\
\text { Total }\end{array}$ & Weight & $\begin{array}{l}\text { Odds Ratio } \\
\text { M-H. Fixed. } 95 \% \mathrm{CI} \text { Year }\end{array}$ & $\begin{array}{c}\text { Odds Ratio } \\
\text { M.H. Fixed. } 95 \% \mathrm{Cl}\end{array}$ \\
\hline Broch & 17 & 82 & 19 & 95 & $9.3 \%$ & $1.05[0.50,2.18] 1998$ & \\
\hline Wong & 31 & 84 & 16 & 57 & $8.0 \%$ & $1.50[0.72,3.10] 2000$ & - \\
\hline Globocnik-petrowic & 39 & 124 & 25 & 80 & $13.9 \%$ & $1.01[0.55,1.85] 2003$ & \\
\hline Santos & 24 & 99 & 22 & 111 & $10.5 \%$ & $1.29[0.67,2.49] 2003$ & \\
\hline Murata & 78 & 188 & 43 & 92 & $22.5 \%$ & $0.81[0.49,1.33] 2004$ & $=$ \\
\hline Liu & 15 & 56 & 27 & 91 & $10.0 \%$ & $0.87[0.41,1.82] 2004$ & \\
\hline Ezzidi & 77 & 383 & 54 & 473 & $25.7 \%$ & $1.95[1.34,2.85] 2009$ & $\rightarrow-$ \\
\hline Total $(95 \% \mathrm{CI})$ & & 1016 & & 999 & $100.0 \%$ & $1.27[1.02,1.57]$ & \\
\hline Total events & 281 & & 206 & & & & \\
\hline \multicolumn{7}{|c|}{$\begin{array}{l}\text { Heterogeneity: } \mathrm{Chi}^{2}=10.13, \mathrm{df}=6(\mathrm{P}=0.12) ; \mathrm{I}^{2}=41 \% \\
\text { Test for overall effect: } Z=2.16(P=0.03)\end{array}$} & 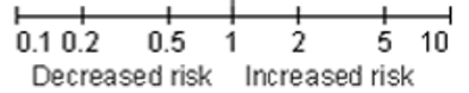 \\
\hline
\end{tabular}


for $4 \mathrm{G} / 4 \mathrm{G}+4 \mathrm{G} / 5 \mathrm{G}$ versus $5 \mathrm{G} / 5 \mathrm{G}: P=0.771$ ). Figure 6 shows the shapes of the funnel plots of $4 \mathrm{G} / 4 \mathrm{G}$ versus $5 \mathrm{G} /$ $5 \mathrm{G}$ overall.

\section{Discussion}

Unraveling the genes that contribute to the pathogenic risk of DR has been one of the major foci of basic research in DR over the past few decades. A large number of putative genes and genetic variants have been reported to be associated with higher risk of DR. A genome-wide association study performed on MexicanAmericans found several SNPs and genes associated with severe DR. None of these loci have been previously linked to DR or diabetes itself [31]. Another genome-wide association study on Taiwanese populations identified five loci not previously associated with DR susceptibility in T2D [32]. This suggests that, until now, no genes have achieved widespread acceptance as conferring high risk of DR in patients with T2D [33]. After a review of the meta-analyses of DR-related gene polymorphisms, only the C677T polymorphism in the methylenetetrahydrofolate reductase gene was detected to moderately augment the risk of DR in T2D overall [34,35]. The Gly82Ser polymorphism in the receptor for advanced glycation end products gene might be considered a risk factor for DR in Asian populations. Moderate evidence was founded for a correlation between the angiotensin-converting enzyme gene and proliferative DR [36]. However, neither the angiotensin-converting enzyme gene insertion/deletion [36-38] nor the vascular endothelial growth factor $-634 \mathrm{C} / \mathrm{G}$ gene [39] showed a significant relationship with $\mathrm{DR}$, either overall or in ethnicity subgroups in meta-analysis so far.

The results of this meta-analysis suggest that PAI-1 4G/ $4 \mathrm{G}$ polymorphism was overall marginally significantly associated with DR risk in T2D. In the stratified analysis, significant associations were observed with Caucasian ethnicity, diabetes duration longer than 10 years and hospital-based studies. To the best of our knowledge, this is the first meta-analysis assessing the association between PAI-1 gene polymorphism and DR.

The earliest investigation into $P A I-1$ polymorphism and DR risk, reported by Nagi et al., revealed a positive relationship between the 4G allele of PAI-1 [19] and DR risk in Pima Indians, whose incidence of diabetes, particularly non-insulin-dependent diabetes, was extremely high

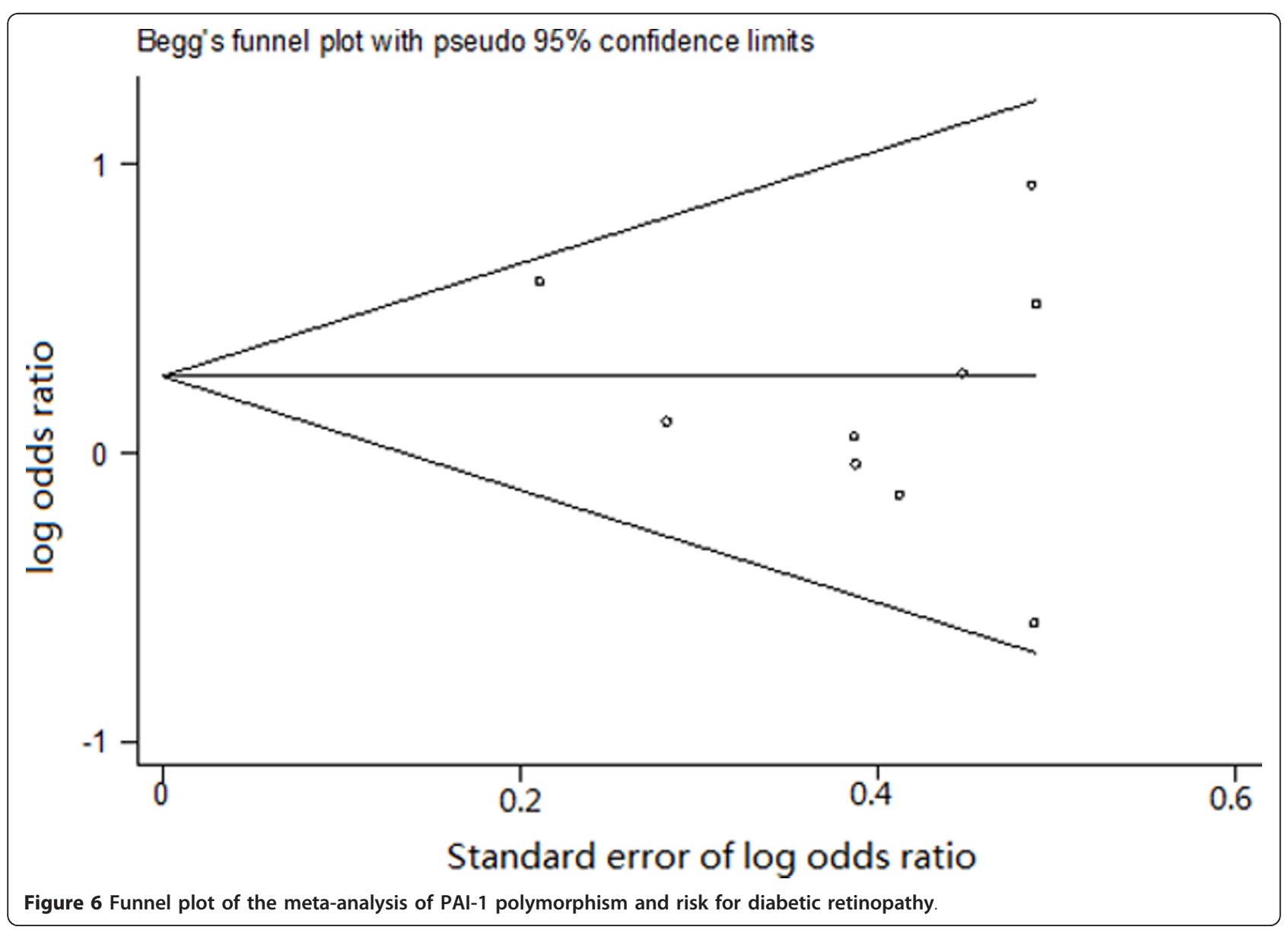


$[40,41]$. However, in the subsequent studies in Caucasian populations, a trend of studies with a lack of association was suggested $[16,18,20,21]$. But in a recent larger casecontrol study in Tunisia that contained a total of 856 adult patients with T2D, Ezzidi et al. reported a significantly higher frequency of the 4G/4G genotype (OR 1.64, 95\%CI 1.10 to 2.43), indicating 4G/4G in PAI-1 locus as a risk factor for DR [17]. All the studies in East Asian populations showed no relationship between 4G/5G polymorphism and DR risk [22-24]. Our meta-analysis confirmed that the 4G/4G genotype of the PAI-1 carried more risk in Caucasian but not Asian participants, even though the overall effect was positive. The differences in ethnic backgrounds, lifestyle, nutrition and living environment may partly explain this discrepancy [42]. We also found a marginally significant susceptibility to DR of T2D between PAI-1 4G/5G polymorphism to the population with longer duration of T2D. This subgroup analysis manifested a gene-environment interaction and highlighted the need for implementing rigorous case-selective criterion in future studies.

We also observed inconsistent results between hospitalbased studies and population-based studies, which may be explained by the biases in hospital-based studies. Control cases in hospital-based studies may be less representative of the general population than those from populationbased studies. Genes do not work in isolation; instead, complex molecular networks and cellular pathways are often involved in disease susceptibility [43]. Taking into account that DR is a complex disease with multifactorial, polygenic and environmental influences, a minor contributing pathogenic role of the PAI-1 polymorphism in specific cases in DR and in co-operation with other factors cannot be totally excluded.

Several potential limitations existed in our meta-analysis and our results should be interpreted with caution. First, as no correction for multiple testing was performed in this meta-analysis, false positive results may have been induced in some fraction because of the application of multiple statistical tests, which would increase the probability of type I errors. Second, our meta-analysis is based on unadjusted estimates because of a lack of original data. For example, the accurate disease time-course of individual patients was unavailable, which may potentially have affected the results where our classification criterion was according to the mean value of the diabetes duration. Third, this metaanalysis was limited by the small sample size - especially in subgroup analysis - though the Egger's test gave no publication bias [44]. Fourth, the existing studies lacked information about potential gene-gene interactions. Last, genotyping methods were different among selected studies, which might affect results. This discrepancy indicates the need to implement rigorous quality control procedures in future studies.

\section{Conclusions}

Our meta-analysis suggests that PAI-1 polymorphism may be associated with elevated DR risk in patients with $\mathrm{T} 2 \mathrm{D}$, especially in the Caucasian population and in patients who have had diabetes for longer than 10 years. Future larger scale epidemiological investigation of this topic should be conducted to validate our findings.

\section{Abbreviations}

Cls: confidence intervals; DR: diabetic retinopathy; DWR: diabetes without DR; ORs: odds ratios; PAl-1: plasminogen activator inhibitor-1; PCR: polymerase chain reaction; SNP: single nucleotide polymorphism; T2D: type 2 diabetes.

\section{Authors' contributions}

KXZ contributed to the idea and design of this study and revised the manuscript. TYZ and CP carried out the screening procedure, performed the statistical analysis and drafted the manuscript. NDL participated in the design of the study, helped performed the statistical analysis and revised the manuscript. EZ helped to improve the English language and gave some suggestions to this manuscript. All authors read and approved the final manuscript.

\section{Competing interests}

The authors declare that they have no competing interests.

\section{Acknowledgements}

This work was supported by the Major And Key Programs of Chinese National Natural Science Foundation (30730099) and 'Project 211' - The Innovation Fund For Graduate Students of Tianjin Medical University (2009GSI20).

\section{Author details}

${ }^{1}$ Tianjin Medical University, 22 Qixiangtai Road, Tianjin, 300020, PR China. ${ }^{2}$ Tianjin Eye Hospital, Tianjin Key Laboratory of Ophthalmology and Visual Science, Tianjin Eye Institute, Clinical College of Ophthalmology Tianjin Medical University, 4 Gansu Road, Tianjin, 300020, PR China. ${ }^{3}$ Department of Ophthalmology, Medical School of Yale University, 300 George Street, New Haven, 06511, USA. ${ }^{4}$ Department of Thoracic Surgery, Tianjin Cancer Institute and Hospital, Huanhu West Road, Tianjin, 300060, PR China. ${ }^{5}$ Saybrook College, Yale University, 242 Elm Street, New Haven, 06511, USA.

Received: 17 May 2012 Accepted: 2 January 2013

Published: 2 January 2013

\section{References}

1. Hallman DM, Huber JC Jr, Gonzalez VH, Klein BE, Klein R, Hanis CL: Familial aggregation of severity of diabetic retinopathy in Mexican Americans from Starr County, Texas. Diabetes Care 2005, 28:1163-1168.

2. Uhlmann K, Kovacs P, Boettcher Y, Hammes HP, Paschke R: Genetics of diabetic retinopathy. Exp Clin Endocrinol Diabetes 2006, 114:275-294.

3. Cilensek I, Mankoc S, Globocnik-Petrovic M, Petrovic D: The 4a/4a genotype of the VNTR polymorphism for endothelial nitric oxide synthase (eNOS) gene predicts risk for proliferative diabetic retinopathy in Slovenian patients (Caucasians) with type 2 diabetes mellitus. Mol Biol Rep 2012, 39:7061-7067.

4. Petrovic MG, Cilensek I, Petrovic D: Manganese superoxide dismutase gene polymorphism (V16A) is associated with diabetic retinopathy in Slovene (Caucasians) type 2 diabetes patients. Dis Markers 2008, 24:59-64.

5. Churchill AJ, Carter JG, Ramsden C, Turner SJ, Yeung A, Brenchley PE, Ray DW: VEGF polymorphisms are associated with severity of diabetic retinopathy. Invest Ophthalmol Vis Sci 2008, 49:3611-3616.

6. Hudson BI, Stickland MH, Futers TS, Grant PJ: Effects of novel polymorphisms in the RAGE gene on transcriptional regulation and their association with diabetic retinopathy. Diabetes 2001, 50:1505-1511.

7. Richeti F, Noronha RM, Waetge RT, de Vasconcellos JP, de Souza OF, Kneipp B, Assis N, Rocha MN, Calliari LE, Longui CA, Monte O, de Melo MB: Evaluation of $A C(n)$ and $C(-106) T$ polymorphisms of the aldose 
reductase gene in Brazilian patients with DM1 and susceptibility to diabetic retinopathy. Mol Vis 2007, 13:740-745.

8. Maeda M, Yamamoto I, Fukuda M, Nishida M, Fujitsu J, Nonen S, Igarashi T, Motomura T, Inaba M, Fujio Y, Azuma J: MTHFR gene polymorphism as a risk factor for diabetic retinopathy in type 2 diabetic patients without serum creatinine elevation. Diabetes Care 2003, 26:547-548.

9. Sobrin L, Green T, Sim X, Jensen RA, Tai ES, Tay WT, Wang JJ, Mitchell P, Sandholm N, Liu Y, Hietala K, lyengar SK, Brooks M, Buraczynska M, van Zuydam N, Smith AV, Gudnason V, Doney AS, Morris AD, Leese GP, Palmer CN, Swaroop A, Taylor HA Jr, Wilson JG, Penman A, Chen CJ, Groop PH, Saw SM, Aung $T$, Klein BE, et al: Candidate gene association study for diabetic retinopathy in persons with type 2 diabetes: the Candidate gene Association Resource (CARe). Invest Ophthalmol Vis Sci 2011, 52:7593-7602

10. Grant MB, Spoerri PE, Player DW, Bush DM, Ellis EA, Caballero S, Robison WG: Plasminogen activator inhibitor (PAI)-1 overexpression in retinal microvessels of PAl-1 transgenic mice. Invest Ophthalmol Vis Sci 2000, 41:2296-2302.

11. Margaglione M, Cappucci G, d'Addedda M, Colaizzo D, Giuliani N, Vecchione G, Mascolo G, Grandone E, Di Minno G: PAl-1 plasma levels in a general population without clinical evidence of atherosclerosis: relation to environmental and genetic determinants. Arterioscler Thromb Vasc Biol 1998, 18:562-567.

12. Ashton $\mathrm{N}$ : Vascular basement membrane changes in diabetic retinopathy. Montgomery lecture, 1973. Br J Ophthalmol 1974, 58:344-366.

13. Henry M, Tregouet DA, Alessi MC, Aillaud MF, Visvikis S, Siest G, Tiret L, Juhan-Vague I: Metabolic determinants are much more important than genetic polymorphisms in determining the PAI- 1 activity and antigen plasma concentrations: a family study with part of the Stanislas Cohort. Arterioscler Thromb Vasc Biol 1998, 18:84-91.

14. Stegnar M, Uhrin P, Peternel P, Mavri A, Salobir-Pajnic B, Stare J, Binder BR: The $4 \mathrm{G} / 5 \mathrm{G}$ sequence polymorphism in the promoter of plasminogen activator inhibitor-1 (PAl-1) gene: relationship to plasma PAl-1 level in venous thromboembolism. Thromb Haemost 1998, 79:975-979.

15. Eriksson P, Kallin B, van 't Hooft FM, Bavenholm P, Hamsten A: Allelespecific increase in basal transcription of the plasminogen-activator inhibitor 1 gene is associated with myocardial infarction. Proc Natl Acad Sci USA 1995, 92:1851-1855

16. Santos KG, Tschiedel B, Schneider J, Souto K, Roisenberg I: Diabetic retinopathy in Euro-Brazilian type 2 diabetic patients: relationship with polymorphisms in the aldose reductase, the plasminogen activator inhibitor-1 and the methylenetetrahydrofolate reductase genes. Diabetes Res Clin Pract 2003, 61:133-136

17. Ezzidi I, Mtiraoui N, Chaieb M, Kacem M, Mahjoub T, Almawi WY: Diabetic retinopathy, PAl-1 4G/5G and -844G/A polymorphisms, and changes in circulating PAI-1 levels in Tunisian type 2 diabetes patients. Diabetes Metab 2009, 35:214-219.

18. Globocnik-Petrovic M, Hawlina M, Peterlin B, Petrovic D: Insertion/deletion plasminogen activator inhibitor 1 and insertion/deletion angiotensinconverting enzyme gene polymorphisms in diabetic retinopathy in type 2 diabetes. Ophthalmologica 2003, 217:219-224.

19. Nagi DK, McCormack L, Mohamed-Ali V, Yudkin JS, Knowler WC, Grant PJ: Diabetic retinopathy, promoter (4G/5G) polymorphism of PAl-1 gene, and PAI-1 activity in Pima Indians with type 2 diabetes. Diabetes Care 1997, 20:1304-1309.

20. Zietz B, Buechler C, Drobnik W, Herfarth H, Scholmerich J, Schaffler A: Allelic frequency of the PAI-1 4G/5G promoter polymorphism in patients with type 2 diabetes mellitus and lack of association with PAI-1 plasma levels. Endocr Res 2004, 30:443-453.

21. Broch M, Gutierrez C, Aguilar C, Simon I, Richart C, Vendrell J: Genetic variation in promoter $(4 \mathrm{G} / 5 \mathrm{G})$ of plasminogen activator inhibitor 1 gene in type 2 diabetes. Absence of relationship with microangiopathy. Diabetes Care 1998, 21:463.

22. Murata M, Maruyama T, Suzuki Y, Saruta T, Ikeda Y: Paraoxonase $1 \mathrm{Gln} /$ Arg polymorphism is associated with the risk of microangiopathy in Type 2 diabetes mellitus. Diabet Med 2004, 21:837-844.

23. Wong TY, Poon P, Szeto CC, Chan JC, Li PK: Association of plasminogen activator inhibitor-1 4G/4G genotype and type 2 diabetic nephropathy in Chinese patients. Kidney Int 2000, 57:632-638.

24. Liu SQ, Xue YM, Yang GC, He FY, Zhao XS: Relationship between plasminogen activator inhibitor-1 gene $4 \mathrm{G} / 5 \mathrm{G}$ polymorphism and type 2 diabetic nephropathy in Chinese Han patients in Guangdong Province. Di Yi Jun Yi Da Xue Xue Bao 2004, 24:904-907.

25. Lau J, loannidis JP, Schmid CH: Quantitative synthesis in systematic reviews. Ann Intern Med 1997, 127:820-826.

26. DerSimonian R, Laird N: Meta-analysis in clinical trials. Control Clin Trials 1986, 7:177-188.

27. Mantel N, Haenszel W: Statistical aspects of the analysis of data from retrospective studies of disease. J Natl Cancer Inst 1959, 22:719-748.

28. Begg CB, Mazumdar M: Operating characteristics of a rank correlation test for publication bias. Biometrics 1994, 50:1088-1101.

29. Egger M, Davey Smith G, Schneider M, Minder C: Bias in meta-analysis detected by a simple, graphical test. BMJ 1997, 315:629-634

30. Moher D, Liberati A, Tetzlaff J, Altman DG: Preferred reporting items for systematic reviews and meta-analyses: the PRISMA statement. BMJ 2009, 339:b2535

31. Fu YP, Hallman DM, Gonzalez VH, Klein BE, Klein R, Hayes MG, Cox NJ, Bell Gl, Hanis CL: Identification of diabetic retinopathy genes through a genome-wide association study among Mexican-Americans from Starr County, Texas. J Ophthalmol 2010, 2010:861291.

32. Huang YC, Lin JM, Lin HJ, Chen CC, Chen SY, Tsai CH, Tsai FJ: Genomewide association study of diabetic retinopathy in a Taiwanese population. Ophthalmology 2011, 118:642-648

33. Liew $G$, Klein $R$, Wong TY: The role of genetics in susceptibility to diabetic retinopathy. Int Ophthalmol Clin 2009, 49:35-52.

34. Niu W, Qi Y: An updated meta-analysis of methylenetetrahydrofolate reductase gene $677 \mathrm{C} / \mathrm{T}$ polymorphism with diabetic nephropathy and diabetic retinopathy. Diabetes Res Clin Pract 2012, 95:110-118.

35. Zintzaras E, Chatzoulis DZ, Karabatsas CH, Stefanidis I: The relationship between C677T methylenetetrahydrofolate reductase gene polymorphism and retinopathy in type 2 diabetes: a meta-analysis. J Hum Genet 2005, 50:267-275.

36. Zhou JB, Yang JK: Angiotensin-converting enzyme gene polymorphism is associated with proliferative diabetic retinopathy: a meta-analysis. Acta Diabetol 2010, 47:187-193.

37. Fujisawa T, Ikegami H, Kawaguchi Y, Hamada Y, Ueda H, Shintani M, Fukuda M, Ogihara T: Meta-analysis of association of insertion/deletion polymorphism of angiotensin l-converting enzyme gene with diabetic nephropathy and retinopathy. Diabetologia 1998, 41:47-53.

38. Wiwanitkit $V$ : Angiotensin-converting enzyme gene polymorphism is correlated to diabetic retinopathy: a meta-analysis. J Diabetes Complications 2008, 22:144-146.

39. Zhao T, Zhao J: Association between the $-634 \mathrm{C} / \mathrm{G}$ polymorphisms of the vascular endothelial growth factor and retinopathy in type 2 diabetes: a meta-analysis. Diabetes Res Clin Pract 2010, 90:45-53.

40. Knowler WC, Bennett PH, Bottazzo GF, Doniach D: Islet cell antibodies and diabetes mellitus in Pima Indians. Diabetologia 1979, 17:161-164.

41. Knowler WC, Bennett PH, Hamman RF, Miller M: Diabetes incidence and prevalence in Pima Indians: a 19-fold greater incidence than in Rochester, Minnesota. Am J Epidemiol 1978, 108:497-505.

42. Hirschhorn JN, Lohmueller K, Byrne E, Hirschhorn K: A comprehensive review of genetic association studies. Genet Med 2002, 4:45-61.

43. Schadt EE: Molecular networks as sensors and drivers of common human diseases. Nature 2009, 461:218-223.

44. Ioannidis JP, Trikalinos TA, Ntzani EE, Contopoulos-loannidis DG: Genetic associations in large versus small studies: an empirical assessment. Lancet 2003, 361:567-571.

\section{Pre-publication history}

The pre-publication history for this paper can be accessed here: http://www.biomedcentral.com/1741-7015/11/1/prepub

doi:10.1186/1741-7015-11-1

Cite this article as: Zhang et al.: Plasminogen activator inhibitor-1 4G/ $5 \mathrm{G}$ polymorphism and retinopathy risk in type 2 diabetes: a metaanalysis. BMC Medicine 2013 11:1. 